\title{
Finite-time cluster synchronization for time-varying delayed complex dynamical networks via hybrid control
}

\author{
Feng Xiao', Qintao Gan ${ }^{1 *}$ (D) and Quan Yuan ${ }^{1}$
}

"Correspondence:

ganqintao@sina.com

${ }^{1}$ Shijiazhuang Campus, Army

Engineering University,

Shijiazhuang, P.R. China

\section{Springer}

\begin{abstract}
Throughout this article, the conundrum on finite-time cluster synchronization is investigated for time-varying delayed complex dynamical networks using a kind of new hybrid control scheme. In the light of Lyapunov stability theorem and finite-time control theory, the finite-time cluster synchronization criteria can be achieved. Besides, we introduce the distinction between cluster synchronization and complete synchronization, which is how to select the controlling nodes. We discuss the differences among cluster synchronization with time-varying delays, complete synchronization with time-varying delays, cluster synchronization with a single delay, and cluster synchronization without delay, which is on constructing Lyapunov functional and designing the finite-time hybrid controllers. Finally, numerical simulations are presented to demonstrate the availability of the theoretical consequences.
\end{abstract}

Keywords: Complex networks; Cluster synchronization; Hybrid control; Time-varying delays; Finite-time synchronization

\section{Introduction}

In the last few years, it is well known that the synchronization problem of complex networks $[1,2]$ has captured much more attention from researchers at various fields, such as physical science, natural science, mathematics, communication, and engineering. Moreover, a multitude of latent applications of synchronization in different engineering domains have been discovered, for instance, image processing systems [3], biological systems [4], and secure communications [5]. Hence, many diverse kinds of synchronization have been examined, incorporating cluster synchronization [6-11], projective synchronization [12-14], lag synchronization [15], generalized synchronization [16], complete synchronization [17], anticipating synchronization [18], phase synchronization [19], etc.

In the real world, on account of specific goals, many biological, social, and technological networks are functionally divided into several groups (also called communities or clusters), and the nodes belonging to the same group reach complete synchronization, but there is no synchronization between any two different groups, which is called cluster synchronization. Owing to its essentiality in communication engineering and biological sci-

(c) The Author(s) 2019. This article is distributed under the terms of the Creative Commons Attribution 4.0 International License (http://creativecommons.org/licenses/by/4.0/), which permits unrestricted use, distribution, and reproduction in any medium, provided you give appropriate credit to the original author(s) and the source, provide a link to the Creative Commons license, and indicate if changes were made. 
ences, much achievement has recently been devoted to studying the cluster synchronization problem of complex dynamical networks, and many remarkable consequences have been established [20-22].

Therefore, cluster synchronization is investigated in this article, which means that nodes can achieve synchronization in each identical group; however, no synchronization arises among nodes in diverse communities. To be more specific, if the nodes can be separated into $n$ nonempty groups, namely

$$
\{1,2, \ldots, N\}=G_{1} \cup G_{2} \cup \ldots \cup G_{m},
$$

where $G_{1}=\left\{1,2, \ldots, c_{1}\right\}, G_{2}=\left\{c_{1}+1, \ldots, c_{2}\right\}, \ldots, G_{m}=\left\{c_{m-1}+1, \ldots, N\right\}$, then the nodes can be synchronized in the uniform community, but no synchronization appears among the unlike communities. When $n=1$, among others, the cluster synchronization is equivalent to the complete synchronization.

For the past few years, progressive control methods, such as pinning control $[6-9,15]$, adaptive control [23], impulsive control [24, 25], intermittent control [6, 9, 25, 26], and so on, have been proposed to accomplish the synchronization of a complex network. In particular, pinning control is one of the valid schemes in the cluster synchronization control of a complex network, which is economy, simplicity, and practicality. However, as far as we know, it is very tough to accomplish the finite-time cluster synchronization. Therefore, in this paper, by using a pinning control scheme for reference, a kind of new finite-time hybrid controller has been projected to command the complex networks and accomplish the finite-time cluster synchronization.

It is well known that time delay $[6-9,11,23,25,27]$ is a highly widespread phenomenon in actual complex network systems. Accordingly, it is essential to investigate the incidence of time delay on the cluster synchronization of a complex network. Ma and Lu [7] inquired into the cluster synchronization of complex network models without time delay. The cluster synchronization of linearly coupled systems was examined by Liu et al. [6] with just a single time delay.

In realistic situations, nevertheless, much intricate and changeable information exchange in complex network is subsistent and time delay is not simply like $x_{i}(t-\tau)$. For this reason, Wang et al. [8] investigated the cluster synchronization of a dynamical complex network with time-varying delays [9], which is more close to actuality. Throughout this paper, the complex networks model is also used for reference, see Wang et al. [8]. However, the cluster synchronization of a complex network is frequently accomplished in finite time $[13,25,26,28-33]$ in practical application. Namely, characters are expected to achieve the cluster synchronization of time-delay complex network as quickly as feasible in reality and the purpose of finite-time synchronization is the optimality in convergence time. It is remarkable to investigate the finite-time cluster synchronization of a complex network in practice. Therefore, many scientific and technical works have been joining the studies for finite-time synchronization of complex networks. Nevertheless, Wang et al. [8] just investigated asymptotic stability of the cluster synchronization. Jiang et al. [13] investigated cluster general projective synchronization of complex networks in finite time without time delay.

Hence, enlightened by the above papers, in this article we aim to achieve the finite-time cluster synchronization of complex networks with time-varying delays via hybrid control. 


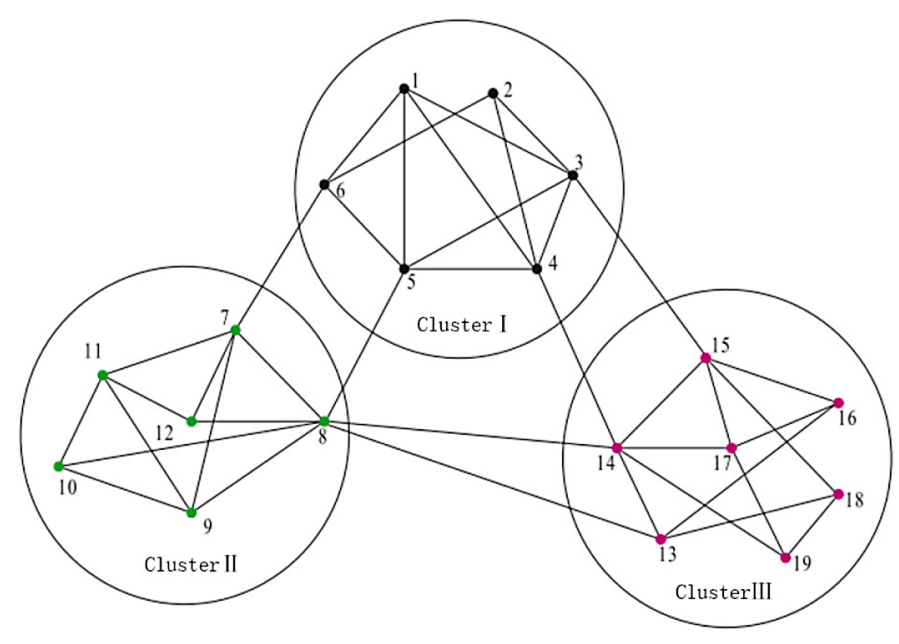

Figure 1 A BA scale-free network with three clusters consisting of 19 nodes

By precedence controlling some key nodes in the $\phi_{i}$ th cluster, which has direct connection with the nodes in other clusters, some sufficient conditions are derived to guarantee the cluster synchronization of networks in finite time as shown in Fig. $1[13,14]$, where the key controlling nodes are Nos. 3, 4, 5, 6, 7, 8, 13, 14, 15.

The work is organized as follows. In Sect. 2, the complex network model is presented, together with some necessary assumptions and lemmas. In Sect. 3, some sufficient criteria for finite-time cluster synchronization are given, and controlled-nodes schemes which comprise undirected network and directed network are proposed in the remark. Numerical simulations are shown in Sect. 4 to check the effectiveness of the theoretical consequences. Ultimately, the paper is concluded in Sect. 5.

\section{Problem description}

In this section, we think about an undirected complex network composed of $N$ nodes with each being an $n$-dimensional dynamical system. The complex dynamical network is depicted by

$$
\begin{aligned}
\dot{x}_{i}(t)= & f_{\phi_{i}}\left(t, x_{i}(t), x_{i}\left(t-\tau_{\phi_{i}}(t)\right)\right)+c_{1} \sum_{j=1}^{N} a_{i j} \Gamma_{1} x_{j}(t) \\
& +c_{2} \sum_{j=1}^{N} b_{i j} \Gamma_{2} x_{j}\left(t-\tau_{\phi_{i}}(t)\right),
\end{aligned}
$$

where $x_{i}(t)=\left(x_{i 1}(t), x_{i 2}(t), \ldots, x_{i n}(t)\right)^{T} \in \mathbf{R}^{n}$ is the state vector of node $i, i=1,2, \ldots, N$. $f_{\phi_{i}}: \mathbf{R} \times \mathbf{R}^{n} \times \mathbf{R}^{n} \rightarrow \mathbf{R}^{n}$ depicts the dynamics of nodes in the $\phi_{i}$ th cluster. $\tau_{\phi_{i}}(t)$ represents time-varying delays in the complex network. $c_{1}>0$ and $c_{2}>0$ are coupling strength. $A=\left(a_{i j}\right)_{N \times N}\left(B=\left(b_{i j}\right)_{N \times N}\right)$ is the outer-coupling configuration matrix that stands for the complex network topological structure. Provided there is a connection from $j$ to node $i(j \neq i)$, then $a_{i j}=a_{j i}>0\left(b_{i j}=b_{j i}>0\right)$, or else $a_{i j}=a_{j i}=0\left(b_{i j}=b_{j i}=0\right)$. The diagonal entries 
of matrices meet the diffusive coupling conditions as follows:

$$
a_{i i}=-\sum_{j=1, j \neq i}^{N} a_{j i} \quad\left(b_{i i}=-\sum_{j=1, j \neq i}^{N} b_{j i}\right) .
$$

$\Gamma_{1} \in \mathbf{R}^{n \times n}$ and $\Gamma_{2} \in \mathbf{R}^{n \times n}$ describe the inner-coupling matrices among the clusters.

The complex network has $m(2 \leq m \leq N)$ clusters and $\{1,2, \ldots, N\}=G_{1} \cup G_{2} \cup \ldots \cup G_{m}$. Provided node $i$ is geared to the $j$ th cluster, then let $\phi_{i}=j$. Utilize $f_{i}(\cdot)$ to stand for the partial dynamics of all nodes in the $i$ th cluster. Let $s_{i}(t)$ be the solution of the network system $\dot{s}_{\phi_{i}}(t)=f_{\phi_{i}}\left(t, s_{i}(t), s_{i}\left(t-\tau_{\phi_{i}}(t)\right)\right)(i=1,2, \ldots, m)$ with $\lim _{t \rightarrow \infty}\left\|s_{i}(t)-s_{j}(t)\right\| \neq 0(i \neq j)$.

The controlled complex network in regard to the complex system (2) can be depicted as follows:

$$
\begin{aligned}
\dot{x}_{i}(t)= & f_{\phi_{i}}\left(t, x_{i}(t), x_{i}\left(t-\tau_{\phi_{i}}(t)\right)\right)+c_{1} \sum_{j=1}^{N} a_{i j} \Gamma_{1} x_{j}(t) \\
& +c_{2} \sum_{j=1}^{N} b_{i j} \Gamma_{2} x_{j}\left(t-\tau_{\phi_{i}}(t)\right)+u_{i}(t), \quad i=1,2, \ldots, N,
\end{aligned}
$$

where $u_{i}(t)(i=1,2, \ldots, N)$ represents the hybrid controllers.

Define the error variables as follows:

$$
e_{i}(t)=x_{i}(t)-s_{\phi_{i}}(t), \quad i=1,2, \ldots, N
$$

where $\dot{s}_{\phi_{i}}(t)=f_{\phi_{i}}\left(t, s_{i}(t), s_{i}\left(t-\tau_{\phi_{i}}(t)\right)\right)$ symbolizes the local dynamics of the nodes in the $\phi_{i}$ th group. Hence, when $\lim _{t \rightarrow t_{1}}\left\|e_{i}(t)\right\|=0(i=1,2, \ldots, N)$, the complex network (4) can achieve finite-time cluster synchronization.

Next, some indispensable assumptions and lemmas are given to demonstrate our primary consequences.

Assumption 1 ([8]) Assume that there exist constants $\zeta_{\phi_{i}}>0$ and $\theta_{\phi_{i}}>0$ for $\forall x, y \in \mathbf{R}^{n}$ and $t \geq 0$ such that the vector-valued function $f_{\phi_{i}}\left(t, x_{i}(t), x_{i}\left(t-\tau_{\phi_{i}}(t)\right)\right)$ satisfies the semiLipschitz condition:

$$
\begin{aligned}
\left(x_{i}(t)-y_{i}(t)\right)^{\mathrm{T}}\left(f_{\phi_{i}}\left(t, x_{i}(t), x_{i}\left(t-\tau_{\phi_{i}}(t)\right)\right)-f_{\phi_{i}}\left(t, y_{i}(t), y_{i}\left(t-\tau_{\phi_{i}}(t)\right)\right)\right) \\
\leq \varsigma_{\phi_{i}}\left(x_{i}(t)-y_{i}(t)\right)^{\mathrm{T}}\left(x_{i}(t)-y_{i}(t)\right)+\theta_{\phi_{i}}\left(x_{i}\left(t-\tau_{\phi_{i}}(t)\right)-y_{i}\left(t-\tau_{\phi_{i}}(t)\right)\right)^{\mathrm{T}} \\
\quad \times\left(x_{i}\left(t-\tau_{\phi_{i}}(t)\right)-y_{i}\left(t-\tau_{\phi_{i}}(t)\right)\right), \quad i=1,2, \ldots, N .
\end{aligned}
$$

Assumption 2 ([8]) Assume that the time-varying delay $\tau_{\phi_{i}}(t)$ is a differential function and $0 \leq \dot{\tau}_{\phi_{i}}(t) \leq \varepsilon \leq 1$.

Lemma 1 ([34]) Suppose that there exist any two vectors $x$ and $y$ and a matrix $S>0$ with appropriate dimensions such that

$$
2 x^{\mathrm{T}} y \leq x^{\mathrm{T}} S x+y^{\mathrm{T}} S^{-1} y .
$$


Lemma 2 ([25]) For $x_{1}, x_{2}, \ldots, x_{n} \in \mathrm{R}^{\mathrm{n}}$, and $0<q<2$ is a real number, the following inequality holds:

$$
\left\|x_{1}\right\|^{q}+\left\|x_{2}\right\|^{q}+\cdots+\left\|x_{n}\right\|^{q} \geq\left(\left\|x_{1}\right\|^{2}+\left\|x_{2}\right\|^{2}+\cdots+\left\|x_{n}\right\|^{2}\right)^{q / 2} .
$$

Lemma 3 ([35]) Assume that there exists an indeterminate vector parameter $\alpha$ which is norm bounded, i.e., $\|\alpha\|<\omega$, where $\omega$ is a known positive invariable, and $\|\cdot\|$ represents matrix 2-norms, then we have $\|\tilde{\alpha}-\alpha\| \leq\|\tilde{\alpha}\|+\|\alpha\| \leq\|\tilde{\alpha}\|+\omega$.

Lemma 4 ([13]) Suppose that a continuous, positive definite function $V(t)$ satisfies the following inequality:

$$
\dot{V}(t) \leq-p V^{\xi}(t), \quad \forall t \geq t_{0}, V\left(t_{0}\right) \geq 0
$$

where $p>0,0<\xi<1$ are two constants. For any given time $t_{0}, V(t)$ satisfies the following inequality: $V^{1-\xi}(t) \leq V^{1-\xi}\left(t_{0}\right)-p(1-\xi)\left(t-t_{0}\right), t_{0} \leq t \leq t_{1}$ and $V(t) \equiv 0, \forall t \geq t^{*}$, with $t^{*}$ given by $t^{*}=t_{0}+V^{1-\xi}\left(t_{0}\right) /[p(1-\xi)]$.

\section{Main results}

In this section, our main results are described to make the complex network (4) realize the finite-time cluster synchronization via pinning control. The synchronization errors are represented by $e_{i}(t)=x_{i}(t)-s_{\phi_{i}}(t)(i=1,2, \ldots, N)$. Then, on the basis of complex network (2), the synchronization error system can be obtained as follows:

$$
\begin{aligned}
\dot{e}_{i}(t)=\tilde{f}_{\phi_{i}}\left(t, x_{i}(t), x_{i}\left(t-\tau_{\phi_{i}}(t)\right)\right)+c_{1} \sum_{j=1}^{N} a_{i j} \Gamma_{1} e_{j}(t) \\
+c_{2} \sum_{j=1}^{N} b_{i j} \Gamma_{2} e_{j}\left(t-\tau_{\phi_{i}}(t)\right)+c_{1} \sum_{j=1}^{N} a_{i j} \Gamma_{1} s_{\phi_{i}}(t) \\
\\
+c_{2} \sum_{j=1}^{N} b_{i j} \Gamma_{2} s_{\phi_{i}}\left(t-\tau_{\phi_{i}}(t)\right)+u_{i}(t), \quad i=1,2, \ldots, N,
\end{aligned}
$$

where $\tilde{f}_{\phi_{i}}\left(t, x_{i}(t), x_{i}\left(t-\tau_{\phi_{i}}(t)\right)\right)=f_{\phi_{i}}\left(t, x_{i}(t), x_{i}\left(t-\tau_{\phi_{i}}(t)\right)\right)-f_{\phi_{i}}\left(t, s_{i}(t), s_{i}\left(t-\tau_{\phi_{i}}(t)\right)\right)(i=$ $1,2, \ldots, N)$. In order to accomplish the cluster synchronization in finite time, the hybrid controllers are contrived as follows:

$$
u_{i}(t)= \begin{cases}-g_{1} e_{i}(t)-\alpha_{i}(t), & i \in \tilde{J}_{\phi_{i}}, \\ -\omega_{i}(t), & i \in J_{\phi_{i}} \tilde{J}_{\phi_{i}},\end{cases}
$$


thereinto

$$
\begin{aligned}
\alpha_{i}(t)= & k \operatorname{sign}\left(e_{i}(t)\right)\left|e_{i}(t)\right|^{\mu} \\
& +k\left(k_{1} \int_{t-\tau_{\phi_{i}}(t)}^{t} e_{i}^{\mathrm{T}}(s) e_{i}(s) \mathrm{d} s\right)^{\frac{1+\mu}{2}} \Psi\left(e_{i}(t),\|e(t)\|\right), \\
\omega_{i}(t)= & k \operatorname{sign}\left(e_{i}(t)\right)\left|e_{i}(t)\right|^{\mu} \\
& +k\left(k_{1} \int_{t-\tau_{\phi_{i}}(t)}^{t} e_{i}^{\mathrm{T}}(s) e_{i}(s) \mathrm{d} s\right)^{\frac{1+\mu}{2}} \Psi\left(e_{i}(t),\|e(t)\|\right) \\
& +2 k\left(g_{1} \int_{t}^{t_{1}} e_{i}^{\mathrm{T}}(s) e_{i}(s) \mathrm{d} s\right)^{\frac{1+\mu}{2}} \Psi\left(e_{i}(t),\|e(t)\|\right),
\end{aligned}
$$

where $i=1,2, \ldots, N ; k$ is a tunable constant, $g_{1}$ is a positive constant denoting the control strength, and $k_{1}$ is a positive constant; if $\|e(t)\| \neq 0, \Psi\left(e_{i}(t),\|e(t)\|\right)=\frac{e_{i}(t)}{\|e(t)\|^{2}}$, or else $\Psi\left(e_{i}(t),\|e(t)\|\right)=0$. Besides, $J_{\phi_{i}}$ represents all the nodes in the $\phi_{i}$ th group and $\tilde{J}_{\phi_{i}}$ denotes the nodes in the $\phi_{i}$ th group which is directly connected with the nodes in other groups.

Remark 1 By using the pinning control method for reference, a finite-time hybrid controller $u_{i}(t)$ has been designed. The structure of the hybrid controller is the same as the one of the pinning controller, i.e., they both divide the nodes into two parts. Thereinto, in $i \in \tilde{J}_{\phi_{i}}$, the nodes are key controlling nodes, which means they will be controlled preferentially. As we all know, the pinning controller is just appropriate for accomplishing asymptotic or exponential synchronization of complex networks. Therefore, there are few literatures to investigate finite-time synchronization by pinning control, not to speak of finite-time cluster synchronization. For the purpose of realizing the finite-time cluster synchronization of complex dynamical networks, the hybrid controller is contrived by us.

Theorem 1 Under Assumption 1, Assumption 2, and Lemma 3, if the following conditions hold:

(1) $\eta_{2}+\frac{1}{2}-\frac{1-\varepsilon}{2} k_{1}<0$,

(2) $\eta_{1}+c_{1} \lambda_{\max }(Q)+\frac{1}{2} c_{2}{ }^{2} \lambda_{\max }\left(P P^{\mathrm{T}}\right)+\frac{1}{2} k_{1}-g_{1}<0$,

where $\eta_{1}=\varsigma_{\phi_{i}}>0, \eta_{2}=\theta_{\phi_{i}}>0, Q=A \otimes \Gamma_{1}$, and $P=B \otimes \Gamma_{2}$, then the complex network (2) under the hybrid controllers (11) can achieve cluster synchronization in finite time:

$$
t_{0} \leq \bar{t} \leq t^{*}=t_{0}+\frac{1}{(1-\mu) k}\left(2 V\left(t_{0}\right)\right)^{\frac{1-\mu}{2}},
$$

where

$$
\begin{aligned}
V\left(t_{0}\right)= & \frac{1}{2} \sum_{i=1}^{N} e_{i}^{2}\left(t_{0}\right)+\frac{1}{2} k_{1} \sum_{i=1}^{N} \int_{t_{0}-\tau_{\phi_{i}}\left(t_{0}\right)}^{t_{0}} e_{i}^{\mathrm{T}}(s) e_{i}(s) \mathrm{d} s \\
& +g_{1} \sum_{\phi_{i}=1}^{m} \sum_{i \in J_{\phi_{i}} \tilde{\nabla}_{\phi_{i}}} \int_{t_{0}}^{t_{1}} e_{i}^{\mathrm{T}}(s) e_{i}(s) \mathrm{d} s .
\end{aligned}
$$

Moreover, $e_{i}\left(t_{0}\right)$ and $\tau_{\phi_{i}}\left(t_{0}\right)$ are original constant values of $e_{i}(t)$ and $\tau_{\phi_{i}}(t)$, respectively. 
Proof A Lyapunov-Krasovskii functional is constructed as indicated below:

$$
\begin{aligned}
V(t)= & \frac{1}{2} \sum_{i=1}^{N} e_{i}^{\mathrm{T}}(t) e_{i}(t)+\frac{1}{2} k_{1} \sum_{i=1}^{N} \int_{t-\tau_{\phi_{i}}(t)}^{t} e_{i}^{\mathrm{T}}(s) e_{i}(s) \mathrm{d} s \\
& +g_{1} \sum_{\phi_{i}=1}^{m} \sum_{i \in J_{\phi_{i}} \tilde{\phi}_{\phi_{i}}} \int_{t}^{t_{1}} e_{i}^{\mathrm{T}}(s) e_{i}(s) \mathrm{d} s .
\end{aligned}
$$

Calculating the derivative of $V(t)$ in relation to time $t$ along the solutions of system (10) outputs the following:

$$
\begin{aligned}
\dot{V}(t)= & \sum_{i=1}^{N} e_{i}^{\mathrm{T}}(t)\left[f_{\phi_{i}}\left(t, x_{i}(t), x_{i}\left(t-\tau_{\phi_{i}}(t)\right)\right)-f_{\phi_{i}}\left(t, s_{i}(t), s_{i}\left(t-\tau_{\phi_{i}}(t)\right)\right)\right. \\
& \left.+c_{1} \sum_{j=1}^{N} a_{i j} \Gamma_{1} e_{j}(t)+c_{2} \sum_{j=1}^{N} b_{i j} \Gamma_{2} e_{j}\left(t-\tau_{\phi_{i}}(t)\right)+u_{i}(t)\right]+\frac{1}{2} k_{1} \sum_{i=1}^{N} e_{i}^{\mathrm{T}}(t) e_{i}(t) \\
& -\frac{1-\dot{\tau}_{\phi_{i}}(t)}{2} k_{1} \sum_{i=1}^{N} e_{i}^{\mathrm{T}}\left(t-\tau_{\phi_{i}}(t)\right) e_{i}\left(t-\tau_{\phi_{i}}(t)\right)-g_{1} \sum_{\phi_{i}=1}^{m} \sum_{i \in J_{\phi_{i}} \tilde{V}_{\phi_{i}}} e_{i}^{\mathrm{T}}(t) e_{i}(t) .
\end{aligned}
$$

Inserting hybrid controllers (11) into $\dot{V}(t)$ and in the light of Assumption 1, we get

$$
\begin{aligned}
\dot{V}(t) \leq & \eta_{1} \sum_{i=1}^{N} e_{i}^{\mathrm{T}}(t) e_{i}(t)+\eta_{2} \sum_{i=1}^{N} e_{i}^{\mathrm{T}}\left(t-\tau_{\phi_{i}}(t)\right) e_{i}\left(t-\tau_{\phi_{i}}(t)\right) \\
& +c_{1} e^{\mathrm{T}}(t)\left(A \otimes \Gamma_{1}\right) e(t)+c_{2} e^{\mathrm{T}}(t)\left(B \otimes \Gamma_{2}\right) e\left(t-\tau_{\phi_{i}}(t)\right) \\
& +\frac{1}{2} k_{1} \sum_{i=1}^{N} e_{i}^{\mathrm{T}}(t) e_{i}(t)-\frac{1-\dot{\tau}_{\phi_{i}}(t)}{2} k_{1} \sum_{i=1}^{N} e_{i}^{\mathrm{T}}\left(t-\tau_{\phi_{i}}(t)\right) e_{i}\left(t-\tau_{\phi_{i}}(t)\right) \\
& -\sum_{\phi_{i}=1}^{m} \sum_{i \in J_{\phi_{i}} \tilde{V}_{\phi_{i}}} g_{1} e_{i}^{\mathrm{T}}(t) e_{i}(t)-\sum_{\phi_{i}=1}^{m} \sum_{i \in \tilde{\phi}_{\phi_{i}}} g_{1} e_{i}^{\mathrm{T}}(t) e_{i}(t)-k \sum_{i=1}^{N} e_{i}^{\mathrm{T}}(t) \\
& \times\left[\left(k_{1} \int_{t-\tau_{\phi_{i}}(t)}^{t} e_{i}^{\mathrm{T}}(s) e_{i}(s) \mathrm{d} s\right)^{\frac{1+\mu}{2}} \Psi\left(e_{i}(t),\|e(t)\|\right)+\operatorname{sign}\left(e_{i}(t)\right)\left|e_{i}(t)\right|^{\mu}\right] \\
& -2 k \sum_{\phi_{i}=1}^{m} \sum_{i \in \delta_{\phi_{i}} \tilde{V}_{\phi_{i}}} e_{i}^{\mathrm{T}}(t)\left[\left(g_{1} \int_{t}^{t_{1}} e_{i}^{\mathrm{T}}(s) e_{i}(s) \mathrm{d} s\right)^{\frac{1+\mu}{2}} \Psi\left(e_{i}(t),\|e(t)\|\right)\right],
\end{aligned}
$$

where $e(t)=\left(\left\|e_{1}(t)\right\|,\left\|e_{2}(t)\right\|, \ldots,\left\|e_{N}(t)\right\|\right)^{\mathrm{T}}$; constants $\eta_{1}>0$ and $\eta_{2}>0$; and $\otimes$ bespeaks the Kronecker product of two matrices.

Let $Q=A \otimes \Gamma_{1}, P=B \otimes \Gamma_{2}$, and from Lemma 1, we have

$$
\begin{aligned}
\dot{V}(t) \leq & \eta_{1} e^{\mathrm{T}}(t) e(t)+\eta_{2} e^{\mathrm{T}}\left(t-\tau_{\phi_{i}}(t)\right) e\left(t-\tau_{\phi_{i}}(t)\right) \\
& +c_{1} e^{\mathrm{T}}(t) Q e(t)+c_{2} e^{\mathrm{T}}(t) P e\left(t-\tau_{\phi_{i}}(t)\right)+\frac{1}{2} k_{1} e^{\mathrm{T}}(t) e(t) \\
& -\frac{1-\dot{\tau}_{\phi_{i}}(t)}{2} k_{1} e^{\mathrm{T}}\left(t-\tau_{\phi_{i}}(t)\right) e\left(t-\tau_{\phi_{i}}(t)\right)-g_{1} e^{\mathrm{T}}(t) e(t)
\end{aligned}
$$


Ciao et al. Advances in Difference Equations

(2019) 2019:93

Page 8 of 21

$$
\begin{aligned}
& -k \sum_{i=1}^{N} e_{i}^{\mathrm{T}}(t)\left[\left(k_{1} \int_{t-\tau_{\phi_{i}}(t)}^{t} e_{i}^{\mathrm{T}}(s) e_{i}(s) \mathrm{d} s\right)^{\frac{1+\mu}{2}} \Psi\left(e_{i}(t),\|e(t)\|\right)\right. \\
& \left.+\operatorname{sign}\left(e_{i}(t)\right)\left|e_{i}(t)\right|^{\mu}\right]-2 k \sum_{\phi_{i}=1}^{m} \sum_{i \in J_{\phi_{i}} \tilde{V}_{\phi_{i}}} e_{i}^{\mathrm{T}}(t) \\
& \times\left[\left(g_{1} \int_{t}^{t_{1}} e_{i}^{\mathrm{T}}(s) e_{i}(s) \mathrm{d} s\right)^{\frac{1+\mu}{2}} \Psi\left(e_{i}(t),\|e(t)\|\right)\right] \\
& \leq \eta_{1} e^{\mathrm{T}}(t) e(t)+\eta_{2} e^{\mathrm{T}}\left(t-\tau_{\phi_{i}}(t)\right) e\left(t-\tau_{\phi_{i}}(t)\right)+c_{1} e^{\mathrm{T}}(t) Q e(t) \\
& +\frac{1}{2} c_{2}{ }^{2} e^{\mathrm{T}}(t) P P^{\mathrm{T}} e(t)+\frac{1}{2} e^{\mathrm{T}}\left(t-\tau_{\phi_{i}}(t)\right) e\left(t-\tau_{\phi_{i}}(t)\right)+\frac{1}{2} k_{1} e^{\mathrm{T}}(t) e(t) \\
& -\frac{1-\dot{\tau}_{\phi_{i}}(t)}{2} k_{1} e^{\mathrm{T}}\left(t-\tau_{\phi_{i}}(t)\right) e\left(t-\tau_{\phi_{i}}(t)\right)-g_{1} e^{\mathrm{T}}(t) e(t)-k \sum_{i=1}^{N} e_{i}^{\mathrm{T}}(t) \\
& \times\left[\left(k_{1} \int_{t-\tau_{\phi_{i}}(t)}^{t} e_{i}^{\mathrm{T}}(s) e_{i}(s) \mathrm{d} s\right)^{\frac{1+\mu}{2}} \Psi\left(e_{i}(t),\|e(t)\|\right)+\operatorname{sign}\left(e_{i}(t)\right)\left|e_{i}(t)\right|^{\mu}\right] \\
& -2 k \sum_{\phi_{i}=1}^{m} \sum_{i \in J_{\phi_{i}} \tilde{V}_{\phi_{i}}} e_{i}^{\mathrm{T}}(t)\left[\left(g_{1} \int_{t}^{t_{1}} e_{i}^{\mathrm{T}}(s) e_{i}(s) \mathrm{d} s\right)^{\frac{1+\mu}{2}} \Psi\left(e_{i}(t),\|e(t)\|\right)\right] \\
& \leq\left(\eta_{2}+\frac{1}{2}-\frac{1-\varepsilon}{2} k_{1}\right) e^{\mathrm{T}}\left(t-\tau_{\phi_{i}}(t)\right) e\left(t-\tau_{\phi_{i}}(t)\right)+\left(\eta_{1}+c_{1} \lambda_{\max }(Q)\right. \\
& \left.+\frac{1}{2} c_{2}{ }^{2} \lambda_{\max }\left(P P^{\mathrm{T}}\right)+\frac{1}{2} k_{1}-g_{1}\right) e^{\mathrm{T}}(t) e(t)-k \sum_{i=1}^{N} e_{i}^{\mathrm{T}}(t) \\
& \times\left[\left(k_{1} \int_{t-\tau_{\phi_{i}}(t)}^{t} e_{i}^{\mathrm{T}}(s) e_{i}(s) \mathrm{d} s\right)^{\frac{1+\mu}{2}} \Psi\left(e_{i}(t),\|e(t)\|\right)+\operatorname{sign}\left(e_{i}(t)\right)\left|e_{i}(t)\right|^{\mu}\right] \\
& -2 k \sum_{\phi_{i}=1}^{m} \sum_{i \in J_{\phi_{i}} \tilde{V}_{\phi_{i}}} e_{i}^{\mathrm{T}}(t)\left[\left(g_{1} \int_{t}^{t_{1}} e_{i}^{\mathrm{T}}(s) e_{i}(s) \mathrm{d} s\right)^{\frac{1+\mu}{2}} \Psi\left(e_{i}(t),\|e(t)\|\right)\right] .
\end{aligned}
$$

According to the conditions of Theorem 1, Lemma 2, and Lemma 3, we can acquire

$$
\begin{aligned}
\dot{V}(t) \leq & -k \sum_{i=1}^{N} e_{i}^{\mathrm{T}}(t)\left[\left(k_{1} \int_{t-\tau_{\phi_{i}}(t)}^{t} e_{i}^{\mathrm{T}}(s) e_{i}(s) \mathrm{d} s\right)^{\frac{1+\mu}{2}} \Psi\left(e_{i}(t),\|e(t)\|\right)\right. \\
& \left.+\operatorname{sign}\left(e_{i}(t)\right)\left|e_{i}(t)\right|^{\mu}\right]-2 k \sum_{\phi_{i}=1}^{m} \sum_{i \in J_{\phi_{i}} \tilde{V}_{\phi_{i}}} e_{i}^{\mathrm{T}}(t) \\
& \times\left[\left(g_{1} \int_{t}^{t_{1}} e_{i}^{\mathrm{T}}(s) e_{i}(s) \mathrm{d} s\right)^{\frac{1+\mu}{2}} \Psi\left(e_{i}(t),\|e(t)\|\right)\right] \\
\leq & -k \sum_{i=1}^{N}\left|e_{i}^{\mathrm{T}}(t) e_{i}(t)\right|^{\frac{1+\mu}{2}}-k \sum_{i=1}^{N}\left(k_{1} \int_{t-\tau_{\phi_{i}}(t)}^{t} e_{i}^{\mathrm{T}}(s) e_{i}(s) \mathrm{d} s\right)^{\frac{1+\mu}{2}} \\
& -2 k \sum_{\phi_{i}=1}^{m} \sum_{i \in J_{\phi_{i}} \tilde{V}_{\phi_{i}}}\left(g_{1} \int_{t}^{t_{1}} e_{i}^{\mathrm{T}}(s) e_{i}(s) \mathrm{d} s\right)^{\frac{1+\mu}{2}}
\end{aligned}
$$




$$
\begin{aligned}
\leq & -2^{\frac{1+\mu}{2}} k\left(\frac{1}{2} \sum_{i=1}^{N} e_{i}^{2}(t)+\frac{1}{2} \sum_{i=1}^{N} k_{1} \int_{t-\tau_{\phi_{i}}(t)}^{t} e_{i}^{\mathrm{T}}(s) e_{i}(s) \mathrm{d} s\right. \\
& \left.+k \sum_{\phi_{i}=1}^{m} \sum_{i \in I_{\phi_{i}} \tilde{V}_{\phi_{i}}} g_{1} \int_{t}^{t_{1}} e_{i}^{\mathrm{T}}(s) e_{i}(s) \mathrm{d} s\right)^{\frac{1+\mu}{2}} \\
= & -2^{\frac{1+\mu}{2}} k V^{\frac{1+\mu}{2}}(t) .
\end{aligned}
$$

On the basis of Lemma 4, for any initial values, the synchronization error system (10) can be obtained by the global stabilization in time $t_{0} \leq \bar{t} \leq t^{*}=t_{0}+\frac{1}{(1-\mu) k}\left(2 V\left(t_{0}\right)\right)^{\frac{1-\mu}{2}}$. When the initial time $t_{0}=0$, we have $t^{*}=\frac{1}{(1-\mu) k}(2 V(0))^{\frac{1-\mu}{2}}$, and we can know that the synchronization time of network counts on value of the constants $k(k>0)$ and $\mu(0 \leq \mu<1)$. Denote $T(k)=$ $\frac{1}{(1-\mu) k}(2 V(0))^{\frac{1-\mu}{2}}$. If we fix the $\mu$, then we get $T^{\prime}(k)=-\frac{1}{(1-\mu) k^{2}}(2 V(0))^{\frac{1-\mu}{2}}<0$; therefore, $T(k)$ is the thoroughly monotone diminishing function. Namely, the more sizeable the tunable constant $k$ is, the shorter time is needed to achieve synchronization. Accordingly, the complex network (2) can achieve the cluster synchronization in finite time $\bar{t}$. This completes the proof.

Remark 2 In this section, we recommended the finite-time hybrid control method to synchronize the complex dynamical networks with time-varying delays. There is a large amount of consequences concerning asymptotic and exponential cluster synchronization via pinning control $[6-9,15]$. Nevertheless, so far as we know, there are few published papers dealing with the finite-time cluster synchronization. And compared with the technique of asymptotic and exponential cluster synchronization for complex dynamical networks with time-varying delays, our consequences are capable of shortening the cluster synchronization time.

In the following, we will give a simple pinning controller to achieve asymptotic cluster synchronization of the complex networks (2) and compare their difference in time of accomplishing synchronization in numerical simulations. The pinning controller is given as follows:

$$
u_{i}^{\prime}(t)= \begin{cases}-g_{1} e_{i}(t), & i \in \tilde{J}_{\phi_{i}}, \\ 0, & i \in J_{\phi_{i}} \tilde{V}_{\phi_{i}},\end{cases}
$$

where $i=1,2, \ldots, N ; g_{1}$ is a positive constant denoting the control strength.

Theorem 2 Suppose that Assumption 1, Assumption 2, and Lemma 3 hold. If the following conditions hold:

(1) $\eta_{2}+\frac{1}{2}-\frac{1-\varepsilon}{2} k_{1}<0$

(2) $\eta_{1}+c_{1} \lambda_{\max }(Q)+\frac{1}{2} c_{2}^{2} \lambda_{\max }\left(P P^{\mathrm{T}}\right)+\frac{1}{2} k_{1}-g_{1}<0$,

where $\eta_{1}=\varsigma_{\phi_{i}}>0, \eta_{2}=\theta_{\phi_{i}}>0, Q=A \otimes \Gamma_{1}$, and $P=B \otimes \Gamma_{2}$, then the complex network (2) under the pinning controller (17) can achieve asymptotic cluster synchronization. 
Proof A Lyapunov-Krasovskii functional is constructed as indicated below:

$$
\begin{aligned}
V(t)= & \frac{1}{2} \sum_{i=1}^{N} e_{i}^{\mathrm{T}}(t) e_{i}(t)+\frac{1}{2} k_{1} \sum_{i=1}^{N} \int_{t-\tau_{\phi_{i}}(t)}^{t} e_{i}^{\mathrm{T}}(s) e_{i}(s) \mathrm{d} s \\
& +g_{1} \sum_{\phi_{i}=1}^{m} \sum_{i \in J_{\phi_{i}} \tilde{V}_{\phi_{i}}} \int_{t}^{t_{1}} e_{i}^{\mathrm{T}}(s) e_{i}(s) \mathrm{d} s .
\end{aligned}
$$

Calculating the derivative of $V(t)$ in relation to time $t$ along the solutions of system (18) outputs the following:

$$
\begin{aligned}
\dot{V}(t)= & \sum_{i=1}^{N} e_{i}^{\mathrm{T}}(t)\left[f_{\phi_{i}}\left(t, x_{i}(t), x_{i}\left(t-\tau_{\phi_{i}}(t)\right)\right)-f_{\phi_{i}}\left(t, s_{i}(t), s_{i}\left(t-\tau_{\phi_{i}}(t)\right)\right)\right. \\
& \left.+c_{1} \sum_{j=1}^{N} a_{i j} \Gamma_{1} e_{j}(t)+c_{2} \sum_{j=1}^{N} b_{i j} \Gamma_{2} e_{j}\left(t-\tau_{\phi_{i}}(t)\right)+u_{i}^{\prime}(t)\right]+\frac{1}{2} k_{1} \sum_{i=1}^{N} e_{i}^{\mathrm{T}}(t) e_{i}(t) \\
& -\frac{1-\dot{\tau}_{\phi_{i}}(t)}{2} k_{1} \sum_{i=1}^{N} e_{i}^{\mathrm{T}}\left(t-\tau_{\phi_{i}}(t)\right) e_{i}\left(t-\tau_{\phi_{i}}(t)\right)-g_{1} \sum_{\phi_{i}=1}^{m} \sum_{i \in J_{\phi_{i}} \tilde{V}_{\phi_{i}}} e_{i}^{\mathrm{T}}(t) e_{i}(t) .
\end{aligned}
$$

The process of proof is similar to that in Theorem 1:

$$
\begin{aligned}
\dot{V}(t) \leq & \eta_{1} \sum_{i=1}^{N} e_{i}^{\mathrm{T}}(t) e_{i}(t)+\eta_{2} \sum_{i=1}^{N} e_{i}^{\mathrm{T}}\left(t-\tau_{\phi_{i}}(t)\right) e_{i}\left(t-\tau_{\phi_{i}}(t)\right) \\
& +c_{1} e^{\mathrm{T}}(t)\left(A \otimes \Gamma_{1}\right) e(t)+c_{2} e^{\mathrm{T}}(t)\left(B \otimes \Gamma_{2}\right) e\left(t-\tau_{\phi_{i}}(t)\right) \\
& +\frac{1}{2} k_{1} \sum_{i=1}^{N} e_{i}^{\mathrm{T}}(t) e_{i}(t)-\frac{1-\dot{\tau}_{\phi_{i}}(t)}{2} k_{1} \sum_{i=1}^{N} e_{i}^{\mathrm{T}}\left(t-\tau_{\phi_{i}}(t)\right) e_{i}\left(t-\tau_{\phi_{i}}(t)\right) \\
& -\sum_{\phi_{i}=1}^{m} \sum_{i \in J_{\phi_{i}} \tilde{V}_{\phi_{i}}} g_{1} e_{i}^{\mathrm{T}}(t) e_{i}(t)-\sum_{\phi_{i}=1}^{m} \sum_{i \in \tilde{I}_{\phi_{i}}} g_{1} e_{i}^{\mathrm{T}}(t) e_{i}(t) \\
\leq & \eta_{1} e^{\mathrm{T}}(t) e(t)+\eta_{2} e^{\mathrm{T}}\left(t-\tau_{\phi_{i}}(t)\right) e\left(t-\tau_{\phi_{i}}(t)\right)+c_{1} e^{\mathrm{T}}(t) Q e(t) \\
& +\frac{1}{2} c_{2}^{2} e^{\mathrm{T}}(t) P P^{\mathrm{T}} e(t)+\frac{1}{2} e^{\mathrm{T}}\left(t-\tau_{\phi_{i}}(t)\right) e\left(t-\tau_{\phi_{i}}(t)\right)+\frac{1}{2} k_{1} e^{\mathrm{T}}(t) e(t) \\
& -\frac{1-\dot{\tau}_{\phi_{i}}(t)}{2} k_{1} e^{\mathrm{T}}\left(t-\tau_{\phi_{i}}(t)\right) e\left(t-\tau_{\phi_{i}}(t)\right)-g_{1} e^{\mathrm{T}}(t) e(t) \\
\leq & \left(\eta_{2}+\frac{1}{2}-\frac{1-\varepsilon}{2} k_{1}\right) e^{\mathrm{T}}\left(t-\tau_{\phi_{i}}(t)\right) e\left(t-\tau_{\phi_{i}}(t)\right) \\
& +\left(\eta_{1}+c_{1} \lambda_{\max }(Q)+\frac{1}{2} c_{2}^{2} \lambda_{\max }\left(P P^{\mathrm{T}}\right)+\frac{1}{2} k_{1}-g_{1}\right) e^{\mathrm{T}}(t) e(t) \\
\leq & 0 .
\end{aligned}
$$

The proof of Theorem 2 is completed. Therefore, the complex network (2) can achieve asymptotic cluster synchronization via the pinning controller (17). 
Remark 3 In the hybrid controller, the methods of selecting key controlling nodes come from the pinning controller. As everyone knows, in the pinning control technique of complex dynamical networks, how to select nodes as pinned nodes is a crucial, significant, and interesting problem. In references [36-38], we can see that when a coupling matrix of nodes is undirected, the authors can choose the highly connected nodes as pinned candidates, and when a coupling matrix of nodes is directed, they select the nodes whose out-degrees are larger than in-degrees as controlled candidates. In this article, the nodes can achieve cluster synchronization in each community by the hybrid control method, whereas no synchronization arises among nodes in varying communities. We make those nodes in the $\phi_{i}$ th group which are directly connected with the nodes in other groups to be key controlling candidates.

Using the method of designing controller and constructing Lyapunov function in this paper, three complex network models derived from system (2) deformation are presented to realize finite-time synchronization respectively.

(1) When the cluster synchronization of complex systems (2) is changed into complete synchronization as indicated below:

$$
\begin{aligned}
\dot{x}_{i}(t)= & f\left(t, x_{i}(t), x_{i}\left(t-\tau_{1}(t)\right)\right)+c_{1} \sum_{j=1}^{N} a_{i j} \Gamma_{1} x_{j}(t) \\
& +c_{2} \sum_{j=1}^{N} b_{i j} \Gamma_{2} x_{j}\left(t-\tau_{2}(t)\right), \quad i=1,2, \ldots, N .
\end{aligned}
$$

Construct the Lyapunov-Krasovskii functional as shown below:

$$
\begin{aligned}
V(t)= & \frac{1}{2} \sum_{i=1}^{N} e_{i}^{\mathrm{T}}(t) e_{i}(t)+\frac{1}{2} k_{1} \sum_{i=1}^{N} \int_{t-\tau_{1}(t)}^{t} e_{i}^{\mathrm{T}}(s) e_{i}(s) \mathrm{d} s \\
& +\frac{1}{2} k_{2} \sum_{i=1}^{N} \int_{t-\tau_{2}(t)}^{t} e_{i}^{\mathrm{T}}(s) e_{i}(s) \mathrm{d} s+g_{1} \sum_{i=l+1}^{N} \int_{t}^{t_{1}} e_{i}^{\mathrm{T}}(s) e_{i}(s) \mathrm{d} s .
\end{aligned}
$$

Therefore, the finite-time hybrid controllers are redesigned as follows:

$$
u_{i}(t)= \begin{cases}-g_{1} e_{i}(t)-\alpha_{i}(t), & 1 \leq i \leq l, \\ -\omega_{i}(t), & l+1 \leq i \leq N\end{cases}
$$

in which

$$
\begin{aligned}
\alpha_{i}(t)= & k \operatorname{sign}\left(e_{i}(t)\right)\left|e_{i}(t)\right|^{\mu}+k \sum_{r=1}^{2}\left(k_{r} \int_{t-\tau_{r}(t)}^{t} e_{i}^{\mathrm{T}}(s) e_{i}(s) \mathrm{d} s\right)^{\frac{1+\mu}{2}} \Psi\left(e_{i}(t),\|e(t)\|\right), \\
\omega_{i}(t)= & k \operatorname{sign}\left(e_{i}(t)\right)\left|e_{i}(t)\right|^{\mu}+k \sum_{r=1}^{2}\left(k_{r} \int_{t-\tau_{r}(t)}^{t} e_{i}^{\mathrm{T}}(s) e_{i}(s) \mathrm{d} s\right)^{\frac{1+\mu}{2}} \Psi\left(e_{i}(t),\|e(t)\|\right) \\
& +2 k\left(g_{1} \int_{t}^{t_{1}} e_{i}^{\mathrm{T}}(s) e_{i}(s) \mathrm{d} s\right)^{\frac{1+\mu}{2}} \Psi\left(e_{i}(t),\|e(t)\|\right) .
\end{aligned}
$$


Moreover, according to the proof of Theorem 1, the following conditions need to be satisfied:

$$
\begin{aligned}
& \text { (1) } \eta_{1}+c_{1} \lambda_{\max }(Q)+\frac{1}{2} c_{2}^{2} \lambda_{\max }\left(P P^{T}\right) \frac{1}{2} k_{1}+\frac{1}{2} k_{2}-g_{1}<0, \\
& \text { (2) } \eta_{2}-\frac{1-\varepsilon_{1}}{2} k_{1}<0, \\
& \text { (3) } \frac{1}{2}-\frac{1-\varepsilon_{2}}{2} k_{2}<0 .
\end{aligned}
$$

Then the complex dynamical networks (21) can achieve complete synchronization in the finite time $t_{1} \leq t_{0}+\frac{1}{(1-\mu) k}\left(2 V\left(t_{0}\right)\right)^{\frac{1-\mu}{2}}$.

(2) When time-varying delays $\tau_{\phi_{i}}(t)$ are reduced to a positive constant $\tau>0$ in complex systems (2) as follows:

$$
\begin{aligned}
\dot{x}_{i}(t)= & f_{\phi_{i}}\left(t, x_{i}(t), x_{i}(t-\tau)\right) c_{1} \sum_{j=1}^{N} a_{i j} \Gamma_{1} x_{j}(t) \\
& +c_{2} \sum_{j=1}^{N} b_{i j} \Gamma_{2} x_{j}(t-\tau), \quad i=1,2, \ldots, N .
\end{aligned}
$$

The Lyapunov-Krasovskii functional is translated into

$$
\begin{aligned}
V(t)= & \frac{1}{2} \sum_{i=1}^{N} e_{i}^{\mathrm{T}}(t) e_{i}(t)+\frac{1}{2} k_{1} \sum_{i=1}^{N} \int_{t-\tau}^{t} e_{i}^{\mathrm{T}}(s) e_{i}(s) \mathrm{d} s \\
& +g_{1} \sum_{\phi_{i}=1}^{m} \sum_{i \in \epsilon_{\phi_{i}} \tilde{V}_{\phi_{i}}} \int_{t}^{t_{1}} e_{i}^{\mathrm{T}}(s) e_{i}(s) \mathrm{d} s .
\end{aligned}
$$

Hence, the finite-time hybrid controllers are redesigned as shown below:

$$
u_{i}(t)= \begin{cases}-g_{1} e_{i}(t)-\alpha_{i}(t), & i \in \tilde{J}_{\phi_{i}}, \\ -\omega_{i}(t), & i \in J_{\phi_{i}} \tilde{V}_{\phi_{i}},\end{cases}
$$

in which

$$
\begin{aligned}
\alpha_{i}(t)= & k \operatorname{sign}\left(e_{i}(t)\right)\left|e_{i}(t)\right|^{\mu}+k\left(k_{1} \int_{t-\tau}^{t} e_{i}^{\mathrm{T}}(s) e_{i}(s) \mathrm{d} s\right)^{\frac{1+\mu}{2}} \Psi\left(e_{i}(t),\|e(t)\|\right), \\
\omega_{i}(t)= & k \operatorname{sign}\left(e_{i}(t)\right)\left|e_{i}(t)\right|^{\mu}+k\left(k_{1} \int_{t-\tau}^{t} e_{i}^{\mathrm{T}}(s) e_{i}(s) \mathrm{d} s\right)^{\frac{1+\mu}{2}} \Psi\left(e_{i}(t),\|e(t)\|\right) \\
& +2 k\left(g_{1} \int_{t}^{t_{1}} e_{i}^{\mathrm{T}}(s) e_{i}(s) \mathrm{d} s\right)^{\frac{1+\mu}{2}} \Psi\left(e_{i}(t),\|e(t)\|\right),
\end{aligned}
$$

and the following conditions need to be satisfied:

(1) $\eta_{1}+c_{1} \lambda_{\max }(Q)+\frac{1}{2} c_{2}^{2} \lambda_{\max }\left(P P^{T}\right)+\frac{1}{2} k_{1}-g_{1}<0$, 
(2) $\eta_{2}+\frac{1}{2}-\frac{1}{2} k_{1}<0$

Then the complex networks (23) can achieve cluster synchronization in the finite time $t_{2} \leq t_{0}+\frac{1}{(1-\mu) k}\left(2 V\left(t_{0}\right)\right)^{\frac{1-\mu}{2}}$.

(3) In case of cluster synchronization without delays, the complex system (2) is translated into

$$
\dot{x}_{i}(t)=f_{\phi_{i}}\left(t, x_{i}(t)\right)+c_{1} \sum_{j=1}^{N} a_{i j} \Gamma_{1} x_{j}(t), \quad i=1,2, \ldots, N
$$

Construct the Lyapunov function as shown below:

$$
V(t)=\frac{1}{2} \sum_{i=1}^{N} e_{i}^{\mathrm{T}}(t) e_{i}(t)+g_{1} \sum_{\phi_{i}=1}^{m} \sum_{i \in J_{\phi_{i}} \tilde{V}_{\phi_{i}}} \int_{t}^{t_{1}} e_{i}^{\mathrm{T}}(s) e_{i}(s) \mathrm{d} s
$$

Hence, the finite-time hybrid controllers are changed into

$$
\begin{aligned}
& u_{i}(t)=\left\{\begin{array}{ll}
-g_{1} e_{i}(t)-\alpha_{i}(t), \quad i \in \tilde{J}_{\phi_{i}}, \\
-\omega_{i}(t),
\end{array} \quad i \in J_{\phi_{i}} \tilde{V}_{\phi_{i}},\right. \\
& \alpha_{i}(t)=k \operatorname{sign}\left(e_{i}(t)\right)\left|e_{i}(t)\right|^{\mu}, \\
& \omega_{i}(t)=k \operatorname{sign}\left(e_{i}(t)\right)\left|e_{i}(t)\right|^{\mu}+2 k\left(g_{1} \int_{t}^{t_{1}} e_{i}^{\mathrm{T}}(s) e_{i}(s) \mathrm{d} s\right)^{\frac{1+\mu}{2}} \Psi\left(e_{i}(t),\|e(t)\|\right),
\end{aligned}
$$

and the following condition needs to be satisfied:

(1) $\eta_{1}+c_{1} \lambda_{\max }(Q)-g_{1}<0$.

Then the complex networks (26) can achieve cluster synchronization in the finite time $t_{3} \leq t_{0}+\frac{1}{(1-\mu) k}\left(2 V\left(t_{0}\right)\right)^{\frac{1-\mu}{2}}$.

Remark 4 From the above consequences, it is clear that the pinning scheme in the hybrid controllers are different between cluster synchronization and complete synchronization. Furthermore, as is well known, the time-varying delays should meet $0 \leq \dot{\tau}_{\phi_{i}}(t) \leq \varepsilon<1$. However, when delay is constant delay, it just satisfies $\tau \geq 0$. Of course, without time delays, constructing a Lyapunov function and designing a finite-time hybrid controller is more simple and brief.

\section{Numerical simulations}

In this section, we show numerical examples to certify the correctness and effectiveness of the proposed finite-time cluster synchronization approach.

In the first instance, consider a delayed neural network with three communities as follows:

$$
\dot{x}(t)=f_{\phi_{i}}\left(t, x(t), x\left(t-\tau_{\phi_{i}}(t)\right)\right), \quad \phi_{i}=1,2,3,
$$


where $x(t)=\left(x_{1}(t), x_{2}(t), x_{3}(t)\right)^{\mathrm{T}}, f_{1}\left(t, x(t), x\left(t-\tau_{1}(t)\right)\right)=D_{1} x(t)+g_{11}(x(t))+g_{12}\left(x\left(t-\tau_{1}(t)\right)\right)$, $f_{2}\left(t, x(t), x\left(t-\tau_{2}(t)\right)\right)=D_{2} x(t)+g_{21}(x(t))+g_{22}\left(x\left(t-\tau_{2}(t)\right)\right)+H$, and $f_{3}\left(t, x_{i}(t), x\left(t-\tau_{1}(t)\right)\right)=$ $D_{3} x(t)+g_{31}(x(t))+g_{32}\left(x\left(t-\tau_{3}(t)\right)\right)$.

In the numerical simulation, we select $g_{11}(x)=\left(0,-x_{1} x_{3}, x_{1} x_{2}\right)^{\mathrm{T}}, g_{12}(x)=\left(0,6 x_{2}, 0\right)^{\mathrm{T}}$, $g_{21}(x)=\left(0,0, x_{1} x_{3}\right)^{\mathrm{T}}, g_{22}(x)=\left(x_{1}, 0,0\right)^{\mathrm{T}}, g_{31}(x)=\left(3.247\left(\left|x_{1}+1\right|-\left|x_{1}-1\right|\right), 0,0\right)^{\mathrm{T}}, g_{32}(x)=$ $\left(0,0,-3.906 \sin \left(0.5 x_{1}\right)\right)^{\mathrm{T}}, H=[0,0,0.2]^{\mathrm{T}}, \tau_{1}(t)=\frac{0.2 e^{t}}{1+e^{t}}, \tau_{2}(t)=\frac{2 e^{t}}{1+e^{t}}, \tau_{3}(t)=\frac{1.2 e^{t}}{1+e^{t}}, D_{1}=$ $\left[\begin{array}{ccc}-10 & 10 & 0 \\ 28 & 4 & 0 \\ 0 & 0 & -\frac{8}{3}\end{array}\right], D_{2}=\left[\begin{array}{ccc}0 & -1 & -1 \\ 1 & 0.2 & 0 \\ 0 & 0 & -1.2\end{array}\right]$ and $D_{3}=\left[\begin{array}{ccc}-2.169 & 10 & 0 \\ 1 & -1 & 1 \\ 0 & -19.53 & -0.1636\end{array}\right]$.

The network model (29) with the above coefficients exhibits chaotic behaviors as shown in Figs. 2-4, with original values $x_{1}(s)=0.2, x_{2}(s)=0.4$, and $x_{3}(s)=0.5, \forall s \in[-2,0]$.

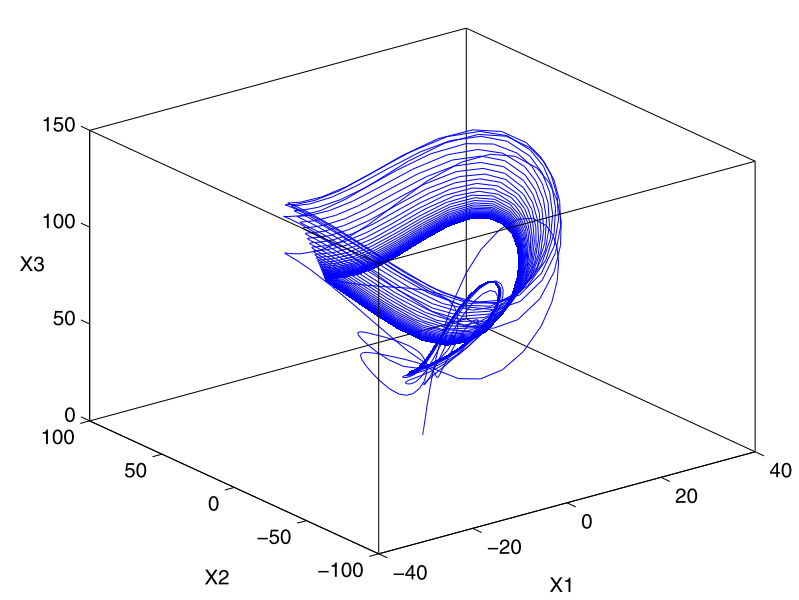

Figure 2 The chaotic state of the first cluster

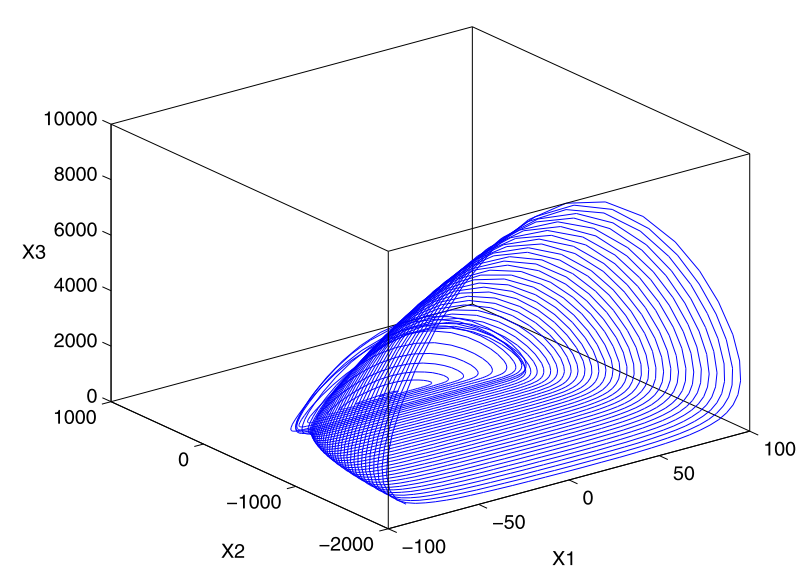

Figure 3 The chaotic state of the second cluster 


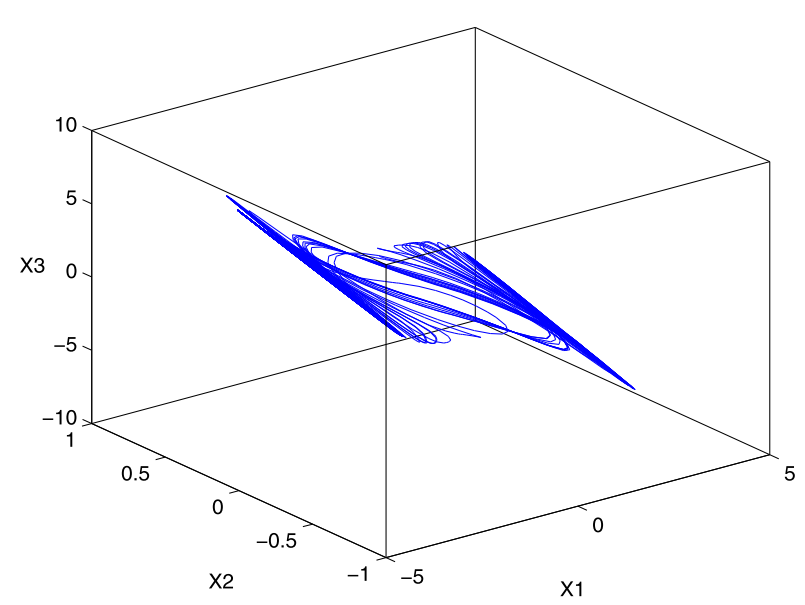

Figure 4 The chaotic state of the third cluster

Then we consider a complex dynamical network with 19 nodes, and there are three groups in Fig. 1. The network is represented as follows:

$$
\begin{aligned}
\dot{x}_{i}(t)= & f_{\phi_{i}}\left(t, x_{i}(t), x_{i}\left(t-\tau_{\phi_{i}}(t)\right)\right)+c_{1} \sum_{j=1}^{N} a_{i j} \Gamma_{1} x_{j}(t) \\
& +c_{2} \sum_{j=1}^{N} b_{i j} \Gamma_{2} x_{j}\left(t-\tau_{\phi_{i}}(t)\right)+u_{i}(t), \quad i=1,2, \ldots, 19
\end{aligned}
$$

where $\Gamma_{1}=\Gamma_{2}=\operatorname{diag}(1,1,1), c_{1}=10, c_{2}=1, \varepsilon=0.1, A=\sum_{j=1}^{N} a_{i j}, B=\sum_{j=1}^{N} b_{i j}, N=19, k=2$, $k_{1}=1.4$, and $g_{1}=41.4$. After a simple calculation, we get $\lambda_{\max }(A)=\lambda_{\max }(B)=-0.0456$, $\lambda_{\max }(Q)=-0.0456, \lambda_{\max }\left(P P^{T}\right)=75.3617, \eta_{1}=3.4707, \eta_{2}=0.1$, and $V(0)=7.677$. The above parameters are substituted into conditions (1) and (2) of Theorem 1, which are met after computing. Next, we can see from Figs. 5-8 that the complex dynamical networks with time-varying delays achieve cluster synchronization in finite time by hybrid control.

The following quantities are applied to measure the course of cluster synchronization:

$$
\begin{aligned}
& \left\{\begin{array}{l}
E_{1}(t)=\sqrt{\sum_{\phi_{i}=1}\left\|x_{i}(t)-s_{1}(t)\right\|^{2}}, \\
E_{2}(t)=\sqrt{\sum_{\phi_{i}=2}\left\|x_{i}(t)-s_{2}(t)\right\|^{2}}, \\
E_{3}(t)=\sqrt{\sum_{\phi_{i}=3}\left\|x_{i}(t)-s_{3}(t)\right\|^{2}},
\end{array}\right. \\
& \begin{cases}E_{12}(t)=\min \left\|x_{i}(t)-x_{j}(t)\right\|, & \phi_{i}=1, \phi_{j}=2, \\
E_{13}(t)=\min \left\|x_{i}(t)-x_{j}(t)\right\|, & \phi_{i}=1, \phi_{j}=3, \\
E_{23}(t)=\min \left\|x_{i}(t)-x_{j}(t)\right\|, & \phi_{i}=2, \phi_{j}=3,\end{cases}
\end{aligned}
$$

where $E_{1}(t), E_{2}(t)$, and $E_{3}(t)$ signify the synchronization errors of each community of the complex networks. $E_{12}(t), E_{13}(t)$, and $E_{23}(t)$ symbolize the errors of the complex networks between two diverse communities. The cluster synchronization is accomplished if the synchronization errors $E_{1}(t), E_{2}(t)$, and $E_{3}(t)$ converge to zero and $E_{12}(t), E_{13}(t)$, and $E_{23}(t)$ do not when $t \rightarrow t_{1}$. As shown in Fig. 5 , it is clearly known that the nodes have achieved finite-time synchronization in the identical group, but there is no synchronization among 

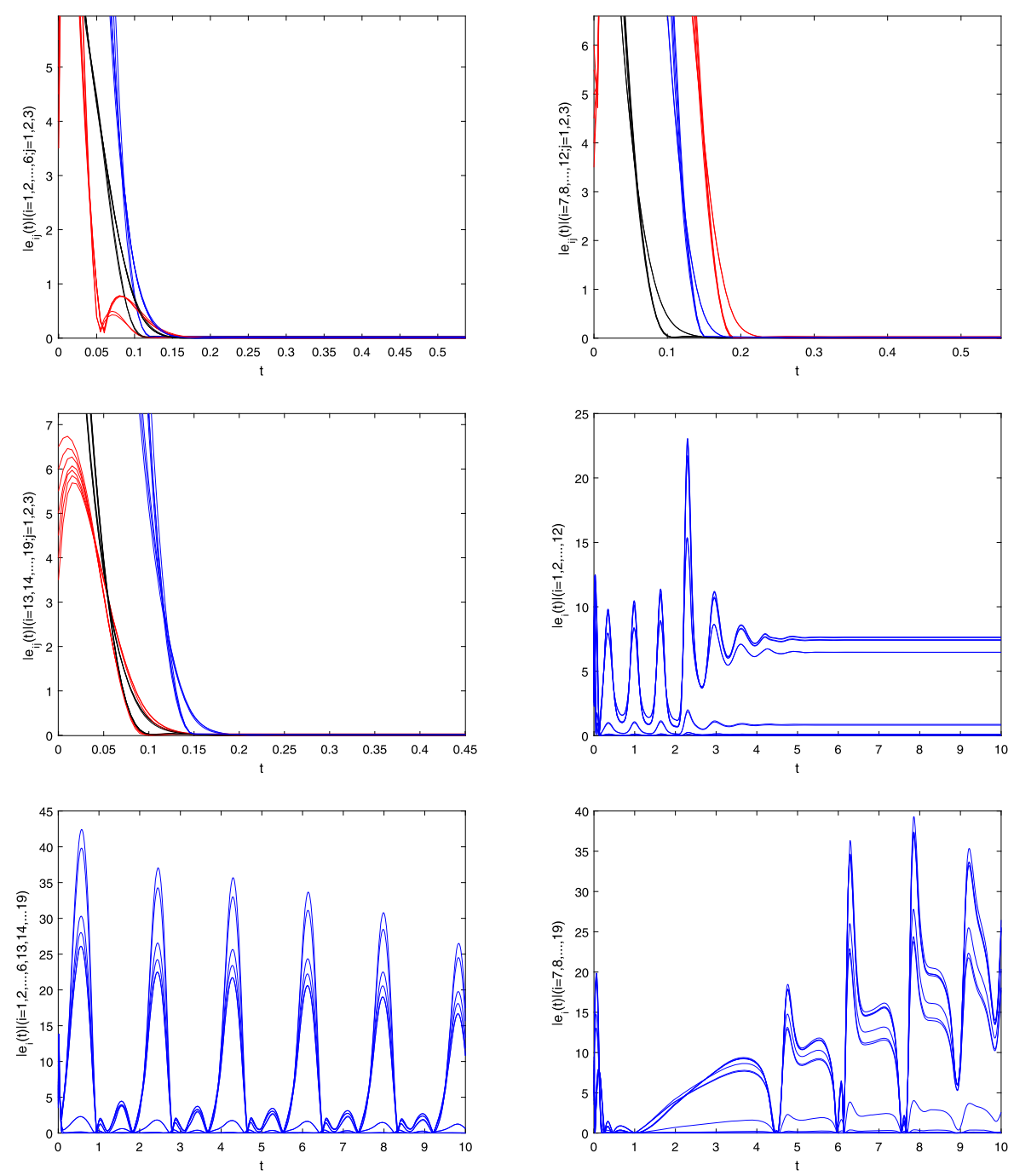

Figure 5 The synchronization errors in the same group and among the different groups

the varying groups. After computing, the finite time satisfies $\bar{t} \leq 1$.959. Figure 6 shows the time evolutions of nodes of each cluster, which also illustrates that the complex networks (4) can achieve cluster synchronization in finite time.

Compared with asymptotic cluster synchronization, the settling time of finite-time cluster synchronization is bounded. It is shown that the convergence rate of finite-time cluster synchronization is faster than that of asymptotic cluster synchronization. As shown in Fig. 7, where line $a$ represents the finite-time synchronization errors and line $b$ represents the asymptotic synchronization errors, line $a$ converges to zero earlier than line $b$.

As pointed out in Sect. 1, for the sake of improving synchronization efficiency, we need to select the nodes in the $\phi$ th group to be the key controlling nodes, which has direct connections with the nodes in other groups. Namely, the key controlling nodes are Nos. $3,4,5,7,8,13,14,15$ in Fig. 1. It is shown that the selection method of controlling nodes is more efficient than another hybrid controller which selects nodes (Nos. 1, 2, 9, 10, 11, $12,16,17,18,19)$ as key controlling nodes. As shown in Fig. 8, where line $a$ represents the 

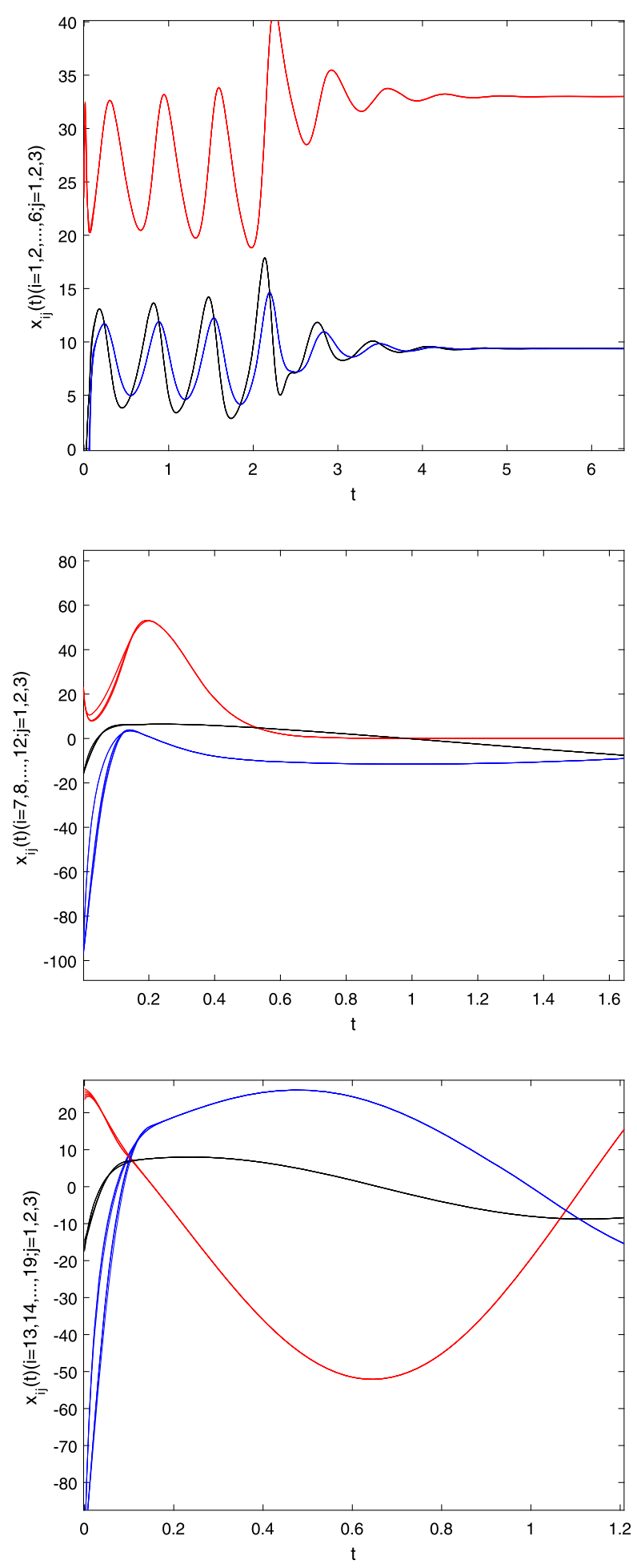

Figure 6 The time evolutions of nodes of three clusters

hybrid controller (11) and line $b$ represents the another hybrid controller, line $a$ converges to zero earlier than line $b$. 

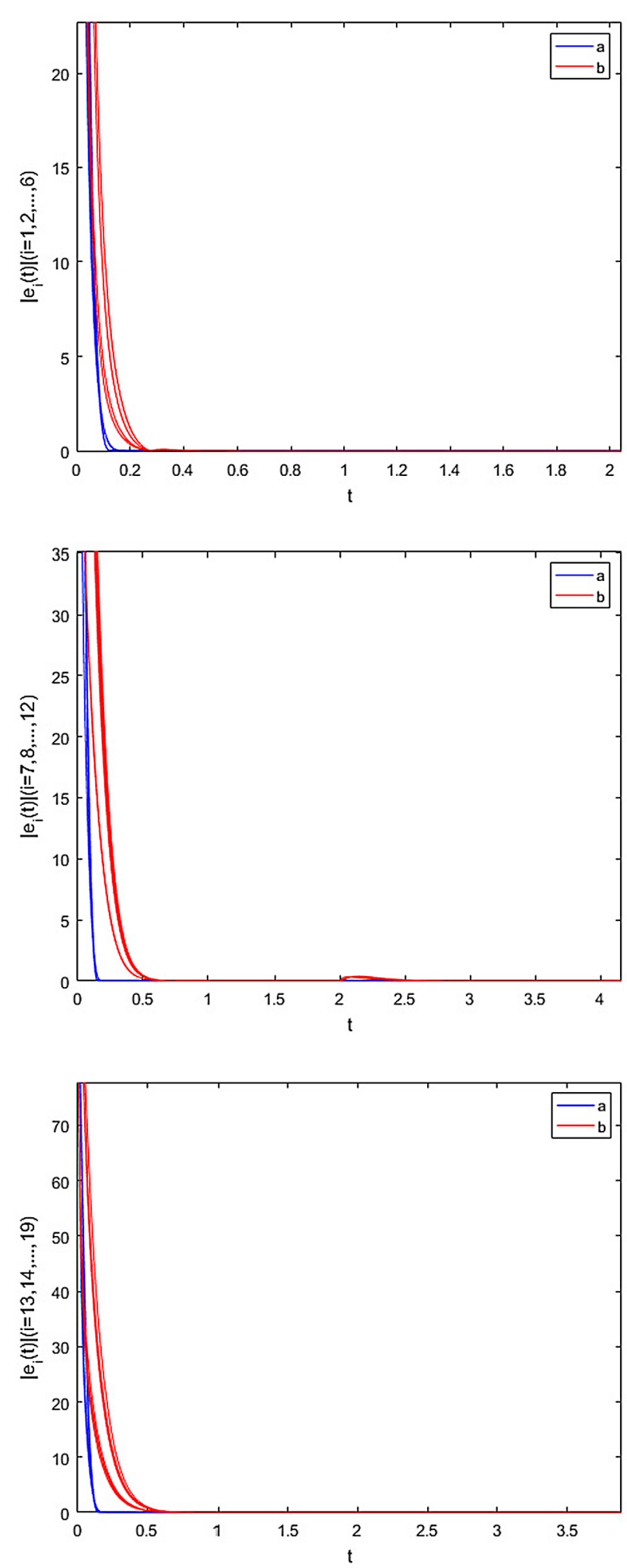

Figure 7 The finite-time synchronization errors and the asymptotic synchronization errors

Remark 5 We can realize that, from the above numerical analysis, our hybrid control method for achieving finite-time cluster synchronization of complex networks model is 

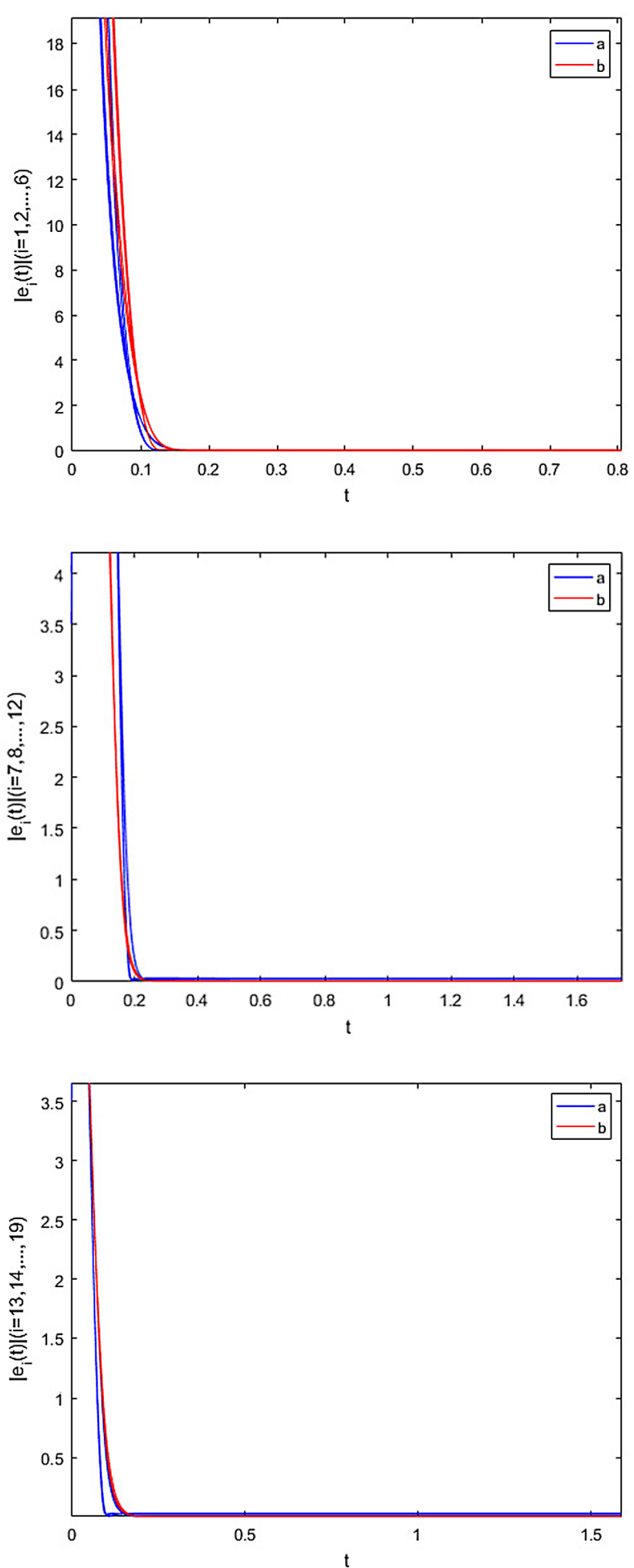

Figure 8 The synchronization errors under different controllers

effective, practical, and economic. In order to achieve cluster synchronization, we first should observe the cluster framework of complex networks. Then, the controller could be 
concluded and designed by this explored cluster structure as well as the controlled nodes could be selected. Finally, the cluster synchronization will be achieved below appropriate coupling strength.

\section{Conclusion}

In this paper, the finite-time cluster synchronization for time-varying delayed complex dynamical networks via hybrid control has been investigated. Sufficient conditions are concluded via formation of the Lyapunov-Krasovskii functional and designing the finitetime hybrid controller with time-varying delays. Hybrid control plans are presented to make the complex dynamical networks achieve cluster synchronization. Eventually, numerical simulations have substantiated the correctness and availability of achieving the finite-time cluster synchronization of complex networks with time-varying delays by the hybrid control technique.

Furthermore, in the coming research, we will consider how to broaden the condition $\varepsilon<1$ in Assumption 2 and investigate the finite-time cluster synchronization with adaptive coupling strength $c(t)$ via intermittent hybrid control.

Funding

This work was supported by the National Natural Science Foundation of China (No. 61305076).

Competing interests

The authors declare that they have no competing interests.

Authors' contributions

The authors contributed equally to the writing of this paper. All authors read and approved the final manuscript.

\section{Publisher's Note}

Springer Nature remains neutral with regard to jurisdictional claims in published maps and institutional affiliations.

Received: 25 October 2018 Accepted: 19 February 2019 Published online: 05 March 2019

References

1. Sun, X., Si, S.: Complex Network Algorithms and Applications. National Defence Industry Press, Beijing (2015)

2. Lu, J., Liu, H., Chen, J.: Synchronization in Complex Dynamical Network. Higher Education Press, Beijing (2016)

3. Wei, G., Jia, Y.: Synchronization-based image edge detection. Europhys. Lett. 59, 814-819 (2002)

4. Wu, Z., Shi, P., Su, H., Chu, J.: Exponential synchronization of neural networks with discrete and distributed delays under time-varying sampling. IEEE Trans. Neural Netw. Learn. Syst. 23, 1368-1376 (2012)

5. Xie, Q., Chen, G., Bollt, E.: Hybrid chaos synchronization and its application in information processing. Math. Comput. Model. 35, 145-163 (2002)

6. Liu, X., Li, P., Chen, T.: Cluster synchronization for delayed complex networks via periodically intermittent pinning control. Neurocomputing 162, 191-200 (2015)

7. Ma, Q., Lu, J: Cluster synchronization for directed complex dynamical networks via pinning control. Neurocomputing $101,354-360(2013)$

8. Wang, S., Yao, H., Zheng, S., Xie, Y.: A novel criterion for cluster synchronization of complex dynamical networks with coupling time-varying delays. Commun. Nonlinear Sci. Numer. Simul. 17, 2997-3004 (2012)

9. Feng, J., Yang, P., Zhao, Y.: Cluster synchronization for nonlinearly time-varying delayed coupling complex networks with stochastic perturbation via periodically intermittent pinning control. Appl. Math. Comput. 291, 52-68 (2016)

10. LV, L., Li, C., Bai, S., Gao, L., Ge, L., Han, C.: Cluster synchronization between uncertain networks with different dynamics. Physica A 468, 429-437 (2017)

11. Li, T., Wang, T., Yang, X., et al.: Pinning cluster synchronization for delayed dynamical networks via Kronecker product. Circuits Syst. Signal Process. 32, 1907-1929 (2013)

12. Du, H., Zeng, Q., Wang, C., et al.: Function projective synchronization in coupled chaotic systems. Nonlinear Anal., Real World Appl. 11, 705-712 (2010)

13. Jiang, S., Cai, G., Cai, S., Tian, L., Lu, X.: Adaptive cluster general projective synchronization of complex dynamic networks in finite time. Commun. Nonlinear Sci. Numer. Simul. 28, 194-200 (2015)

14. $\mathrm{Wu}, \mathrm{Z}$., Fu, X.: Cluster projective synchronization between community networks with nonidentical nodes. Physica A 391, 6190-6198 (2012)

15. Guo, W.: Lag synchronization of complex networks via pinning control. Nonlinear Anal., Real World Appl. 12, 2579-2585 (2011)

16. Liu, H., Chen, J., Lu, J., Cao, M.: Generalized synchronization in complex dynamical networks via adaptive couplings. Physica A 389, 1759-1770 (2010) 
17. Cao, J., Chen, G., Li, P.: Global synchronization in an array of delayed neural networks with hybrid coupling. IEEE Trans. Syst. Man Cybern. B 38, 488-498 (2008)

18. Voss, H.: Anticipating chaotic synchronization. Phys. Rev. E 61, 5115-5119 (2000)

19. Zhou, T., Zhao, M., Chen, G., Yan, G., Wang, B.: Phase synchronization on scale-free networks with community structure. Phys. Lett. A 368, 431-434 (2007)

20. Cao, J. Li, L.: Cluster synchronization in an array of hybrid coupled neural networks with delay. Neural Netw. 22 , 335-342 (2009)

21. Lu, W., Liu, B., Chen, T.: Cluster synchronization in networks of coupled nonidentical dynamical systems. Chaos 20, $013120(2010)$

22. Zhang, J., Ma, Z., Zhang, G.: Cluster synchronization induced by one-node clusters in networks with asymmetric negative couplings. Chaos 23, 043128 (2013)

23. Zheng, S., Wang, S., Dong, G., Bi, Q:: Adaptive synchronization of two nonlinearly coupled complex dynamical networks with delayed coupling. Commun. Nonlinear Sci. Numer. Simul. 17, 284-291 (2012)

24. Sun, W., Hu, T., Chen, Z., Chen, S., Xiao, L.: Impulsive synchronization of a general nonlinear coupled complex network. Commun. Nonlinear Sci. Numer. Simul. 16, 4501-4507 (2011)

25. Mei, J., Jiang, M., Xu, W., Wang, B.: Finite-time synchronization control of complex dynamical networks with time delay. Commun. Nonlinear Sci. Numer. Simul. 18, 2462-2478 (2013)

26. Mei, J., Jiang, M., Wu, Z., Wang, X.: Periodically intermittent controlling for finite-time synchronization of complex dynamical networks. Nonlinear Dyn. 79, 295-305 (2015)

27. Pei, J., Fan, H., Zhao, Y., Feng, J.: Adaptive synchronization of fractional-order nonlinearly coupled complex networks with time delay and external disturbances. IEEE Access 6, 4653-4663 (2018)

28. Xu, C., Yang, X., Lu, J., Feng, J., Alsaadi, F., Hayat, T.: Finite-time synchronization of networks via quantized intermittent pinning control. IEEE Trans. Cybern. 48, 3021-3027 (2018)

29. Zhang, C., Wang, X., Wang, S., Zhou, W., Xia, Z.: Finite-time synchronization for a class of fully complex-valued networks with coupling delay. IEEE Access 6, 17923-17932 (2018)

30. Zheng, M., Li, L., Peng, H., Xiao, J., Yang, Y., Zhao, H.: Finite-time projective synchronization of memristor-based delay fractional-order neural networks. Nonlinear Dyn. 89, 2641-2655 (2017)

31. Qin, X., Wang, C., Li, L., Peng, H., Yang, Y., Ye, L.: Finite-time modified projective synchronization of memristor-based neural network with multi-links and leakage delay. Chaos Solitons Fractals 116, 302-315 (2018)

32. Zheng, M., Li, L., Peng, H., Xiao, J., Yang, Y., Zhang, Y., Zhao, H.: Finite-time stability and synchronization of memristor-based fractional-order fuzzy cellular neural networks. Commun. Nonlinear Sci. Numer. Simul. 69, 272-291 (2018)

33. Zhang, Y., Li, L., Peng, H., Xiao, J., Zheng, M., Zhao, H.: Finite-time synchronization for memristor-based BAM neural networks with stochastic perturbations and time-varying delays. Int. J. Robust Nonlinear Control 28, 5118-5139 (2018)

34. Cai, S., Zhou, P., Liu, Z:: Pinning synchronization of hybrid-coupled directed delayed dynamical network via intermittent control. Chaos 24, 033102 (2014)

35. Jiang, S., Cai, G., Cai, S., Tian, L., Lu, X.: Corrigendum to "Adaptive cluster general projective synchronization of complex dynamic networks in finite time" [28, 1995-200 (2015)]. Commun. Nonlinear Sci. Numer. Simul. 46, 161-163 (2017)

36. Wang, J., Ma, X., Wen, X., Sun, Q.: Pinning lag synchronization of drive-response complex networks via intermittent control with two different switched periods. Physica A 461, 278-287 (2016)

37. Yu, W., Chen, G. L L , J.: On pinning synchronization of complex networks. Automatica 45, 429-435 (2009)

38. Song, Q., Cao, J.: On pinning synchronization of directed and undirected complex dynamical networks. IEEE Trans. Circuits Syst. I 57, 672-680 (2010)

\section{Submit your manuscript to a SpringerOpen ${ }^{\circ}$ journal and benefit from:}

- Convenient online submission

- Rigorous peer review

- Open access: articles freely available online

- High visibility within the field

- Retaining the copyright to your article

Submit your next manuscript at $\gg$ springeropen.com 